\title{
A reflection on success factors in implementing sensory modulation in an acute mental health setting
}

\author{
Tawanda Machingura, Chris Lloyd
}

ensory modulation is an overarching term given to a suite of tools used to assist mental health service users to moderate their responses when distressed (Brown et ảl, 2002; Champagne and Stromberg, 2004). Sensory tools can, for example, be used to help service users who are agitated to become calmer, those who are anxious to feel less so, or help activate service users who are depressed (Champagne, 2011). Clinicians also use sensory modulation to help some service users to lessen feelings of self-harm (Lee et al, 2010; Smith and Jones, 2014) or to manage violent situations (Sutton et al, 2013).

In acute mental health inpatient units, sensory modulation is a clinician-guided intervention that occurs after a sensory assessment has been conducted with a trained clinician (Chalmers et al, 2012; Scanlan and

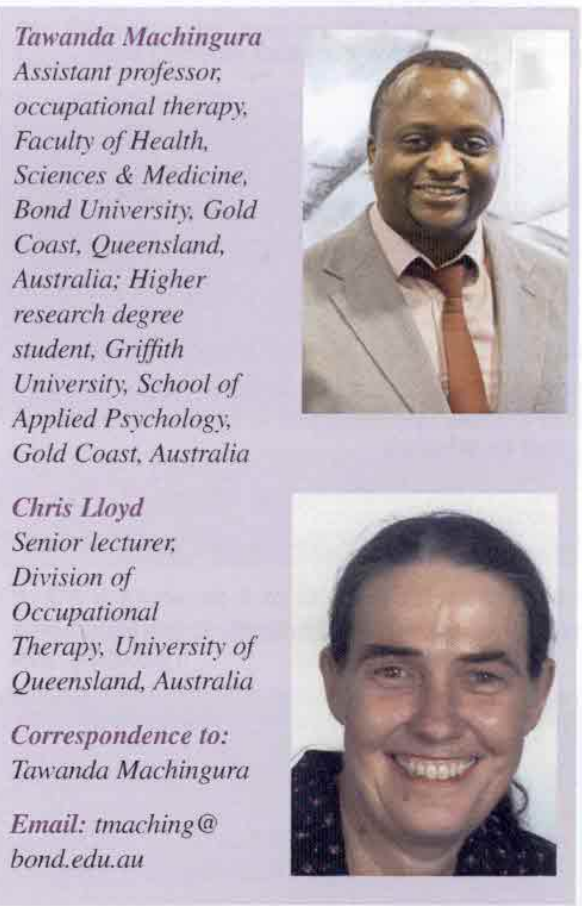

Novak, 2015). Sensory modulation can either be a planned intervention or it can be used 'as necessary'. Sensory modulation most often occurs in a dedicated space called a sensory room (Novak et al, 2012). In sensory rooms, service users are able to choose from a variety of sensory tools. Examples of these tools include audio equipment, soft or pleasant feeling materials, pleasant aromas, electronic massage chairs and weighted blankets for the lap or shoulders that provide a sensation of pressure.

Champagne et al (2010) and Champagne and Koomar (2012) recommend establishing sensory-based approaches in mental health occupational therapy practice. Sensory modulation is the profession of occupational therapy's response to the sociopolitical movement based on increasing demands from service users and governments for service user-centred humane approaches for adults with mental health conditions who present with behavioural issues associated with violent incidents (Champagne, 2011).

The need to reduce seclusion and restraint and to promote the least restrictive trauma informed practice came about as the result of concerns about the use of seclusion and restraint. There is growing research evidence on sensory modulation as an effective intervention for reducing the need for seclusion and restraint (Lloyd et al, 2014; Scanlan and Novak, 2015) particularly in inpatient settings as a component of a larger, multistrategy approach to reducing seclusion and/or restraint.

\section{BACKGROUND}

The implementation of a variety of measures by Gold Coast Hospital and Health Service - coordinated by its Mental Health Seclusion and Restraint Committee with the purpose of reducing seclusion and restraint and promoting the least restrictive trauma informed practice-were evaluated. The specific measures implemented by the Committee to reduce the use of seclusion and restraint in the inpatient units were:

- Having a dedicated seclusion and restraint committee

- Staff education

- Implementation of sensory modulation

- New documentation

- Debriefing and reporting processes.

In addition, two seclusion rooms were redeveloped to a low stimulus area specifically for the use of sensory modulation.

The implementation was coordinated by a working group, called the Sensory Modulation Working Party (SMWP), which was chaired by the assistant director of occupational therapy at the hospital. Membership of the Committee consisted of two senior occupational therapists, two ward-based occupational therapists, a service user/carer representative, the nursing unit managers of the two wards involved and two clinical nurses. This committee was responsible for developing assessment and evaluation tools, procedures or protocols, a staff training programme in sensory modulation, and overseeing the running of the sensory modulation programme on the wards. This paper, however, focuses on the implementation of sensory modulation and staff training and does not cover other measures put in place to reduce seclusion and restraint.

\section{EVALUATION METHODS}

A critical reflection methodology was used, as well as quantitative and qualitative data collected during the implementation of 
sensory modulation in the unit. Several key indicators and evaluation tools were selected to capture outcomes of the sensory modulation programme (Table 1). In this article, we reflect on the implementation using a sensory modulation implementation and reporting checklist (SMIRC) we developed (Table 2 ) based on our experience of implementing sensory modulation.

\section{FINDINGS}

\section{Training}

Monthly sensory modulation training sessions were provided to the ward staff by selected members of the SMWP who had participated in a train the trainer programme. The training was 30 minutes in length and covered the basic concepts of sensory modulation, which included:

- Introduction: why use sensory approaches, why now

n Theory related to sensory modulation: the senses, history, origins, polyvagal theory, sensory processing, arousal and self-regulation, sensory kits and sensory diets

- Practical: identifying and utilising different equipment on oneself and with each other, build awareness of own sensory preferences. Utilise sensory preference screen on oneself

- Orientation to sensory modulation folder

- Before and after survey

- Sensory activity suggestions short list

- Sensory modulation care plan

- Short case scenario

- Sensory preference screen

- All participants were directed to online training ( 2 hours online learning - a statewide course on sensory approaches) if

\section{Table 1. Key indicators and evaluation tools}

Use of sensory room/kits

Use of pro re nata pre and post implementation

Queensland Health state-wide clinical incident management information system: PRIME Clinical Incidents $(\mathrm{Cl})$ rates pre and post implementation

Service user rating scale pre and post implementation

Staff rating scale/feedback pre and post implementation they were interested in pursuing further training.

The goal of the SMWP was to provide sensory modulation training to the majority (or all) of the staff working on the two wards; an adult acute mental health unit and a child and youth acute unit.

Following the first round of training, it was found that a significant number of staff ( $>80 \%$ of the expected 30 staff) had not attended the training. This occurred for a number of reasons, such as being away sick, being on leave and being on night duty. It was then concluded after reviewing the training attendance records that further training and education of staff was required in order to capture a larger number of staff. The intention was to get all unit staff trained so that interventions could be carried out

\section{Table 2. Sensory modulation implementation and reporting checklist}

Time (how much time will be spent and/or was spent on):

Training: Awareness raising and training of staff to become competent in sensory approaches Assessments: For example, completing sensory preference screens Interventions: For example, providing sensory interventions

Evaluation: For example, completing evaluation tools, collating data and writing reports

Volume (who and how many stakeholders were involved and/or will be involved):

Staff: How many staff were or will be involved?

Patients: How many patients were or will be involved?

\section{Costs (how much was spent and/or is needed):}

Equipment: For example, sensory items, rocking chair and massage cushions

Space: For example, converting a seclusion room into a sensory room

Additional staff time: For example, this was cost neutral for us as we utilised existing staff within usual contracted hours

Impact (what was and/or will be the impact):

Local: impact on current local processes and service/team goals/function

Upstream: For example to teams/units where your service users come from

Adjacent: Impact on other units that share staff or resources with your unit or team

Downstream: Impact on teams or units that receive your service users, e.g. continuing care teams or units

\section{Benefits (what were and/or will be the benefits and to whom):}

Distress: Potential to reduce distress in patients

Conflict and containment: Potential to reduce the rates and or severity of conflict and containment

Staff: Potential to boost staff confidence and morale in general and specifically when dealing with distressed patients

Supporting systems (how were and/or will the changes be sustained and supported):

Governance: Sponsor/committees and working groups

Quality and Safety: Procedures/work instructions/guidelines

Communication: Ongoing communication and promotion e.g. reporting, newsletter, intranet 
attended the sensory modulation group went on to actively promote sensory modulation to others in their unit, thereby acting as champions of sensory modulation in their units. This is similar finding to the staff training intervention described by Cook et al (2015), which aimed to change the attitudes, skills and behaviour of staff in a mental health rehabilitation setting.

\section{Evaluation of training}

Training was evaluated using six key questions (Table 3). Participants were asked to rate their knowledge of sensory modulation before commencing the training and then again at the completion of training. They were then asked to rate their confidence in using sensory modulation before commencing the training and again

\section{Table 3. Training evaluation questionnaire}

1a. On a scale of 1 to 5 where 1 is the lowest and 5 highest please rate your knowledge of sensory modulation before commencing the training?

$$
1
$$

2

4

5

1b. Please rate your knowledge of sensory modulation after completion of the training?

1

2

3

4

5

Any additional comments

2a. On a scale of 1 to 5 where 1 is the lowest and 5 highest please rate your confidence to use sensory modulation before commencing the training?

5

2b. Please rate your confidence in using sensory modulation after completion of the training?

1

2

3

4

5

3. On a scale of 1 to 5 where 1 is the lowest and 5 is the highest, how effective overall would you describe the training?

1

5

Why?

4. On a scale of 1 to 5 where 1 is the lowest and 5 is the highest how effective would you rate the facilitators' ability to relay key information?

1

2

3

4

5

Any additional comments

5. On a scale of 1 to 5 where 1 is the lowest and 5 is the highest how applicable and relevant to your daily practice and professional development did you find the training?

1

2

3

Any additional comments

6. Please provide any additional comments on how you think the training may be improved or enhanced all stages of implementation was a critical success factor when implementing sensory modulation in mental health units.

\section{Assessments}

The assessment process included implementation of a sensory screen tool developed by members of the SMWP by adapting the sensory preference screen (Champagne, 2011). The sensory preference screen starts out by looking at the top five calming strategies and the top five alerting strategies. It then goes on to look at what are some of the things that make the person angry, upset or cause them to go into crisis. The next section addresses warning signs and looks at what the person's warning signs are when they feel they may lose control. The screen concludes by looking at crisis prevention strategies or calming preferences that may have helped the person to feel better when having a hard time.

This tool was a mandatory requirement before the commencement of any sensory modulation intervention. The sensory screening tool could be completed by any staff member that had received the sensory modulation training provided by the SMWP.

In the process of assessment or treatment, if any staff member identified that a service user required further, more in-depth assessment, then a referral would be made for an occupational therapy sensory profile assessment. On review we found that $100 \%$ of the sensory preference screens were completed by either occupational therapists or occupational therapy students. This was coincidental in that a new project of student run clinics was started at about the same time as the project.

\section{Interventions}

Most of the individual interventions were completed by nursing staff where as all the group programmes were run by occupational therapists or other allied health professionals. Staff used a rating scale to rate the usefulness of each activity they used with service users. Results indicated that most activities were beneficial to service users. This is a reflection of staff having been trained to tailor interventions according to service users' individual sensory preferences.

\section{Evaluation}

Staff using sensory approaches recorded their data onto prescribed forms as part 
of the everyday note writing, and the evaluation report was completed by the assistant director of occupational therapy, who spent an hour a week on reporting and report writing.

\section{Volume}

This initiative was designed to involve all unit staff and the interventions and assessments to be accessible to all service users. However, we found that only about $70 \%$ of the staff could attend training due to logistical problems such as shift work. We also found that service users were more ready to engage in sensory modulation when they understood what it is. Those service users who attended sensory modulation groups where they learnt about sensory preferences and explored some of the equipment where more likely to participate in sensory modulation interventions than those who did not.

\section{Costs}

Multiple and varied items are used for the sensory modulation rooms, carts and interventions to provide service users access to stimuli in all sensory systems for optimal experiences and outcomes. Examples of items or interventions that could be utilised under each of the sensory systems were also provided (Table 4).

\section{Impact}

The SMWP identified the need for uncomplicated and accessible assessment processes from feedback obtained from unit staff, the majority of whom were nursing staff, as well as published research articles (Champagne, 2011) that support a multidisciplinary team approach of the sensory modulation programme. The process was standardised and included in the sensory modulation procedure.

The intention of the SMWP was to minimise the impact of implementing sensory modulation on the unit's operations. Impact on units and teams that were closely related to the unit such as the emergency department, continuing care teams and the acute care team were minimised by ensuring that the changes would not impact on bed flow by causing delays to the admission or discharge processes. During the evaluation, we received feedback from continuing care staff about how some services users were reporting benefits from using sensory modulation. This information was then used to roll out training to all staff in the service who were interested. Furthermore, sensory information was also utilised in crisis planning and integrated into the admission processes of two units.

\section{Benefits}

We have previously reported the results of introducing sensory modulation to a ward in an effort to reduce seclusion in an earlier publication (Lloyd et al, 2014). The results indicated that seclusion rates have significantly reduced; however, use of pro re nata and seclusion duration times remain unchanged. Sensory room data showed that most service users who have used sensory modulation have reported reduction in distress as measured by pre and post sensory modulation activity ratings. Repeated measures analysis of scores on the Emotions Rating Scale showed a significant decline in severity of emotional distress after use of the sensory modulation room compared with prior to the use of the sensory modulation room $(\mathrm{F}=79.3, \mathrm{df}=1, P<0.001)$. Mean scores fell from 6.58 to 3.72 (mean decline: 2.68 , $95 \%$ confidence interval: $2.22-3.50$ ) and the within subjects effect size was very large (partial eta squared: 0.54).

\section{Supporting systems \\ Governance}

Senior leadership members were involved from the beginning and approval was obtained to start a working party reporting directly to a committee consisting of senior leaders. This meant that support to provide resources and train staff was anticipated and articulated from the beginning of the initiative.

\section{Table 4. Sensory activities used with participants}

\begin{tabular}{l}
$\begin{array}{l}\text { Activities attendees } \\
\text { experienced during } \\
\text { the open day }\end{array} \quad \begin{array}{r}\text { Sensory } \\
\text { system } \\
\text { targeted }\end{array}$ \\
\hline
\end{tabular}

Using isometric exercises

Using a weighted ball/

toy

Using weighted

blankets

\section{Using a rocking chair}

Proprioception

Attendees to experience change in levels of alertness and increase self-regulation

Attendees to experience enhanced grounding

Vestibular

Attendees to experience changes in levels of alertness,

Attendees to experience vestibular stimulation

To increase self-regulation

$\begin{array}{ll}\begin{array}{l}\text { Using a massage chair } \\ \text { Squeezing a stress ball }\end{array} & \begin{array}{c}\text { Tactile } \\ \text { Listening to sounds }\end{array} \\ \begin{array}{l}\text { Listening to music } \\ \text { Auditory }\end{array} & \begin{array}{r}\text { Attendees to experience changes in alertness, } \\ \text { decrease tactile defensiveness and increase } \\ \text { self-regulation } \\ \text { auditory hypersensitivity and to increase } \\ \text { self-regulation }\end{array}\end{array}$

Increasing or
decreasing the
amount of light in the
room
Watching fish in an
aquarium

$\begin{array}{ll}\text { Peppermint } \quad \text { Olfactory } & \text { Attendees to experience a change in olfactory } \\ \text { hyper- or hypo-sensitivity and how to increase }\end{array}$

Scented oils self-regulation

Crunchy foods

Gustatory

\begin{abstract}
Attendees to experience changes in levels of alertness, and how to decrease visual hypersensitivity and increase self-regulation
\end{abstract}




\section{Quality and safety}

The working party was then responsible for engaging with staff and developing procedures that included protocols on safety and precautions, training requirements, use of assessments, use of the room, service user supervision, borrowing guidelines and sign out procedure, and data collection processes. This resulted from the need for specific tasks requiring standardisation in administration and due to the necessary safety and infection control practices. The development of guidelines has also been identified as a need and is currently a work in progress.

\section{Limitations}

This approach to implementing sensory modulation relies heavily on a train the trainer model. We found that not all staff could be reached using this method of training due to shift work and other logistical issues such as having to cancel training when a trainer was not available. We propose to use a blended approach where the option of online self-directed training is made available to staff. This would improve access of training and consistency of training.

The checklist we used for implementation and reporting has not been widely tested and is limited in reliability and validity. We plan to further test and improve the validity and reliability of the SMIRC.

\section{Key practice implications}

Based on our reflections of implementation of sensory modulation using the sensory modulation implementation and reporting checklist (SMIRC) and results reported in detail in Lloyd et al (2014), we concluded that we had successfully implemented sensory modulation in an acute inpatient unit. We felt that the success was in large part due to:

- Use of an implementation and reporting process. We found that using the SMIRC to implement sensory modulation meant that we could anticipate barriers and put in place strategies to ensure that they were avoided or their impact was minimised well in advance.

- Integrated clinical process. Sensory modulation interventions must be seen as a clinical intervention.
This means interventions must be supervised by trained staff, undertaken in a collaborative manner with the service user and be recorded in the organisation's clinical record system. It was also noted that assessment tools used need to be simple and easy to administer, as well as being flexible and sensitive to the needs of the service user. Feedback was received from staff that cited a lack of time for paperwork as an issue.

- Staff training and engagement. Identification of key staff and clarification in regards to the intent. Key staff were identified as being unit nursing staff and it was ensured that they understood the purpose of sensory modulation and their part in the project. Sensory modulation implementation requires ongoing training, a multidisciplinary approach, engagement with all staff at all stages of implementation. In particular, it was found that where nurses become trainers of other nurses the message was much better received.

- Governance. A procedure was developed to guide staff on implementing and using sensory modulation. Protocols for care of equipment were developed. Governance structures were established to assist with obtaining funding and resources. The service was successful in acquiring significant funding to buy equipment and also received some equipment, such as weighted blankets, sourced by a senior manager who had been incorporated into the governance structures.

\section{CONCLUSIONS}

Based on our experience, there are many essential ingredients needed to successfully implement sensory modulation in an acute mental health unit.

Staff training and engagement, clear implementation and reporting protocols, integrated clinical processes and clear supporting systems are all essential ingredients that services implementing sensory modulation should attend in order to increase the likelihood of successful implementation. IJTR
Acknowledgements: We would like to acknowledge members of the SMWP at Gold Coast Hospital and Health Service for their input and feedback. We particularly want to thank Linda Parlato (Occupational Therapist) for her invaluable input in this project. We would also like to thank Gold Coast Health's Mental Health Research Committee for their support.

Brown C, Cromwell RL, Fillion D et al (2002) Sensory processing in schizophrenia: missing and avoiding information. Schizophr Res 55(1-2): 187-195, doi: 10.1016/S09209964(01)00255-9

Chalmers A, Harrison S, Mollison, K et al (2012) Establishing sensory-based approaches in mental health inpatient care: a multidisciplinary approach. Australas Psychiatry 20(1): 35-9. doi: $10.1177 / 1039856211430146$

Champagne $\mathrm{T}$ (2011) The influence of posttraumatic stress disorder, depression, and sensory processing patterns on occupational engagement: A case study. Work 38(1): 67-75

Champagne T, Koomar J (2012) Evaluating sensory processing in mental health occupational therapy practice. OT Practice 17(5): CE1-8

Champagne T, Stromberg N (2004) Sensory approaches in inpatient psychiatric settings: innovative alternatives to seclusion and restraint. J Psychosoc Nurs Ment Health Serv 42(9): 34-44

Champagne T, Koomar J, Olson L (2010) Sensory processing evaluation and intervention in mental health. OT Practice 15(3): CE1-9

Cook S, Mundy T, Killaspy H et al (2015) Development of a staff training intervention for inpatient mental health rehabilitation units to increase service users' engagement in activities. Br J Occup Ther 79(3): 144-52. doi: 10.1177/0308022615600175

Lee SJ, Cox A, Whitecross F et al (2010) Sensory assessment and therapy to help reduce seclusion use with service users needing psychiatric intensive care. J Psychiatric Intensive Care 6(2): 83-90, doi.org/10.1017/S1742646410000014

Lloyd C, King R, Machingura T (2014) An investigation into the effectiveness of sensory modulation in reducing seclusion within an acute mental health unit. Adv Mental Health 12(2): 93-100. doi: 10.1080/18374905.2014.11081887

Novak T, Scanlan J, McCaul D et al (2012) Pilot study of a sensory room in an acute inpatient psychiatric unit. Australas Psychiatry 20(5): 401-6. doi: $10.1177 / 1039856212459585$

Scanlan JN, Novak T (2015) Sensory approaches in mental health: A scoping review. Aust Occup Ther J 62(5): 277-85. doi: 10.1111/14401630.12224

Smith S, Jones J (2014) Use of a sensory room on an intensive care unit. J Psychosoc Nurs Ment Health Serv 52(5): 22-30. doi: 10.3928/02793695-20131126-06

Sutton D, Wilson M, Van Kessel K, Vanderpyl J (2013) Optimizing arousal to manage aggression: A pilot study of sensory modulation. Int J Ment Health Nurs 22(6): 500-11. doi: $10.1111 /$ inm. 12010 